\title{
A Virtual Telescope for the Open University Science Foundation Course
}

\author{
By Andrew J. Norton' ${ }^{1}$ AND Mark H. Jones ${ }^{2}$ \\ ${ }^{1}$ Astronomy Group, Department of Physics, The Open University, \\ Walton Hall, Milton Keynes MK7 6AA \\ ${ }^{2}$ Centre for Educational Software, Academic Computing Service, \\ The Open University, Walton Hall, Milton Keynes MK7 6AA
}

\section{Introduction}

The Open University is the UK's foremost distance teaching university. For over twenty five years we have been presenting courses to students spanning a wide range of degree level and vocational subjects. Since we have no pre-requisites for entry, a major component of our course profile is a selection of foundation courses comprising one each in the Arts, Social Science, Mathematics, Technology and Science faculties. The Science Faculty's foundation course is currently undergoing a substantial revision. The new course, entitled "S103: Discovering Science", will be presented to students for the first time in 1998.

The University has always aimed to make use of appropriate technologies for delivering its teaching material. For the first time, this new version of the Science Foundation Course will make extensive use of fully integrated CD-ROM based activities. One of these is a "Virtual Telescope" package designed to give students an appreciation of what is required to measure the expansion of the Universe.

\section{S103: Discovering Science}

The four science disciplines of biology, chemistry, Earth sciences and physics each contribute in equal measure to the course. Whilst parts of the course are deliberately multi-disciplinary, in order to give students a feel for science as a whole, other parts of the course reflect the very different natures of the four component disciplines. The course will be studied over a thirty-two week period and is accredited at 60 CATS points at level one. (CATS stands for Credit Accumulation Transfer Scheme and is the national scheme within the UK for classifying higher education courses.) A degree is awarded for an accumulation of 360 CATS points, split between levels one, two and three. An outline of the Course is given in Table 1 .

The Course begins with a multi-disciplinary opening that is strongly skills led. After another combined science block which considers the question of 'A Temperate Earth?', the course narrows down to some more discipline specific content. Within 'The Earth and its Place in the Universe' one week out of the four is spent on a Cook's Tour of the Universe, travelling from clusters of galaxies down to the solar system. The remainder of the block covers aspects of Earth science. Following this opening, the students encounter discipline specific blocks in biology ('Unity within Diversity'), physics ('Energy'), and chemistry ('The World and its Atoms'). They then complete their tour from the very large to the very small with two weeks of physics in 'Atomic and Subatomic Structure'. In this final block of the first half of the Course, students learn about electrons in atoms, nuclei in nucleons, and quarks in nucleons from the point of view of a quantum description. This is balanced by a largely experimental section about the dual wave and particle nature of light. 
Table 1. The Structure of S103: Discovering Science

Block Title

1

Taking the world apart..

$2 \quad$ A Temperate Earth?

3 The Earth and its Place in the Universe

4

5

6

7
Unity within Diversity

Energy

The World and its Atoms

Atomic and subatomic Structure
Weeks

2

$\begin{array}{lr}4 & \text { Multi } \\ 4 & \text { Physics / E.Sci } \\ 2 & \text { Biology } \\ 1 & \text { Physics } \\ \mathbf{3} & \text { Chemistry } \\ 2 & \text { Physics }\end{array}$

Putting it back together...

8 Building and Recycling with Atoms

9 Continuity and Change

10 Earth and Life through Time

11 Universal Processes

12 Life in the Universe
Disciplines

\section{Multi}

Multi

/ E.Sci

Biology

Physics

Chemistry

Biology

Earth Sci.

Physics

Multi

With the second half of the Course, students begin to rebuild up to the large scale structure of the Universe. Several weeks of chemistry ('Building and Recycling with Atoms'), biology ('Continuity and Change') and Earth science ('Earth and Life through Time') culminate in another physics block, but this time with a cosmological slant ('Universal Processes'). It is this block that is home to the Virtual Telescope, of which more in a moment. The Course concludes with another multi-disciplinary case study on the theme of 'Life in the Universe'. As can be seen, there are six weeks of study devoted to each of the four science disciplines, coupled with eight weeks of multi-disciplinary activity. Of this, around two-and-a-half weeks of study are on themes that may be broadly classified as Astronomy based.

\section{Course Components}

Each of the twelve blocks of the Course is based around a Book text that is specifically written for the purpose, by authors at the OU. Accompanying this is a Study File that contains notes to the other activities within the Course and provides a place for the students to keep track of their own skills development and learning process.

The other Course components include twelve video programmes, ten broadcast television programmes, about twenty-six CD-ROM based computer activities and eight practical work activities to be carried out at home. The students will attend a one-week residential school which incorporates three full-day laboratory sessions on inter-disciplinary activities, a day-long field trip, a project to be worked on in groups throughout the week, and tutorial sessions each evening. Students are also allocated a personal tutorcounsellor who hosts regional tutorials throughout the year and is available for guidance and support at any time.

Assessment of the course is via eight tutor marked assignments (50\%), plus a centrally marked assignment based on the final block of the Course (10\%), and an end year examination $(40 \%)$. 


\section{Universal Processes}

The penultimate block of the Course is entitled 'Universal Processes', and an outline of its structure is given in Table 2. The block links together the two most exciting areas of modern physics, namely the quest for a theory of everything and an investigation of the early Universe. The particle physics and cosmology are presented in a way that is accessible to the students with minimal mathematics, and yet builds on the background knowledge of subatomic structure and fundamental forces gained earlier in the course.

Table 2. The Structure of Block 11: Universal Processes

\section{Section Title}

1

2

2.2

3

3.1

3.2

3.3

3.4

3.5

4

4.1

4.2

5

5.1
Introduction

The evolving Universe

The expanding Universe

The cooling Universe

Four universal forces

Electromagnetic interactions

Strong interactions

Weak interactions

Gravitational interactions

Four forces in the Sun

Unified theories

Electroweak unification

Further unification?

The history of the Universe

5.2

From big bang to the present

The future

Other components

+CD-ROM

+CD-ROM

+ CD-ROM

+Video

$+\mathrm{TV}$

+CD-ROM

The block begins by investigating the two pieces of evidence which allow us to conclude that we live in an evolving Universe, namely the Hubble expansion and the cosmic microwave background. This is where the 'Virtual Telescope' sits in the Course, as discussed below. After establishing that the Universe is expanding and cooling, the question is posed as to how the hot big bang and the very early Universe can be understood. This requires an appreciation by the students of the four fundamental forces of nature. After these are each described in turn, with the help of further CD-ROM based activities, the processes within the Sun are used as an example of the four forces in action. This section is accompanied by a video programme shot at a solar observatory and a neutrino detector experiment. The unification of these fundamental forces is discussed next, with the help of a TV programme filmed at CERN. The students are now equipped to embark on a tour from the big bang to the present day, and far into the future. Again this is done with the help of a CD-ROM based activity.

\section{A Virtual Telescope}

The aim of this activity is to provide the student with a virtual experiment in which they measure the expansion of the Universe. Whilst the emphasis is on making the experience as 'real' as possible, we are not dealing with astronomy students. So we have no mention of magnitudes when discussing brightness or luminosity, and there are no coordinate positions given in terms of right ascension and declination, for instance. 


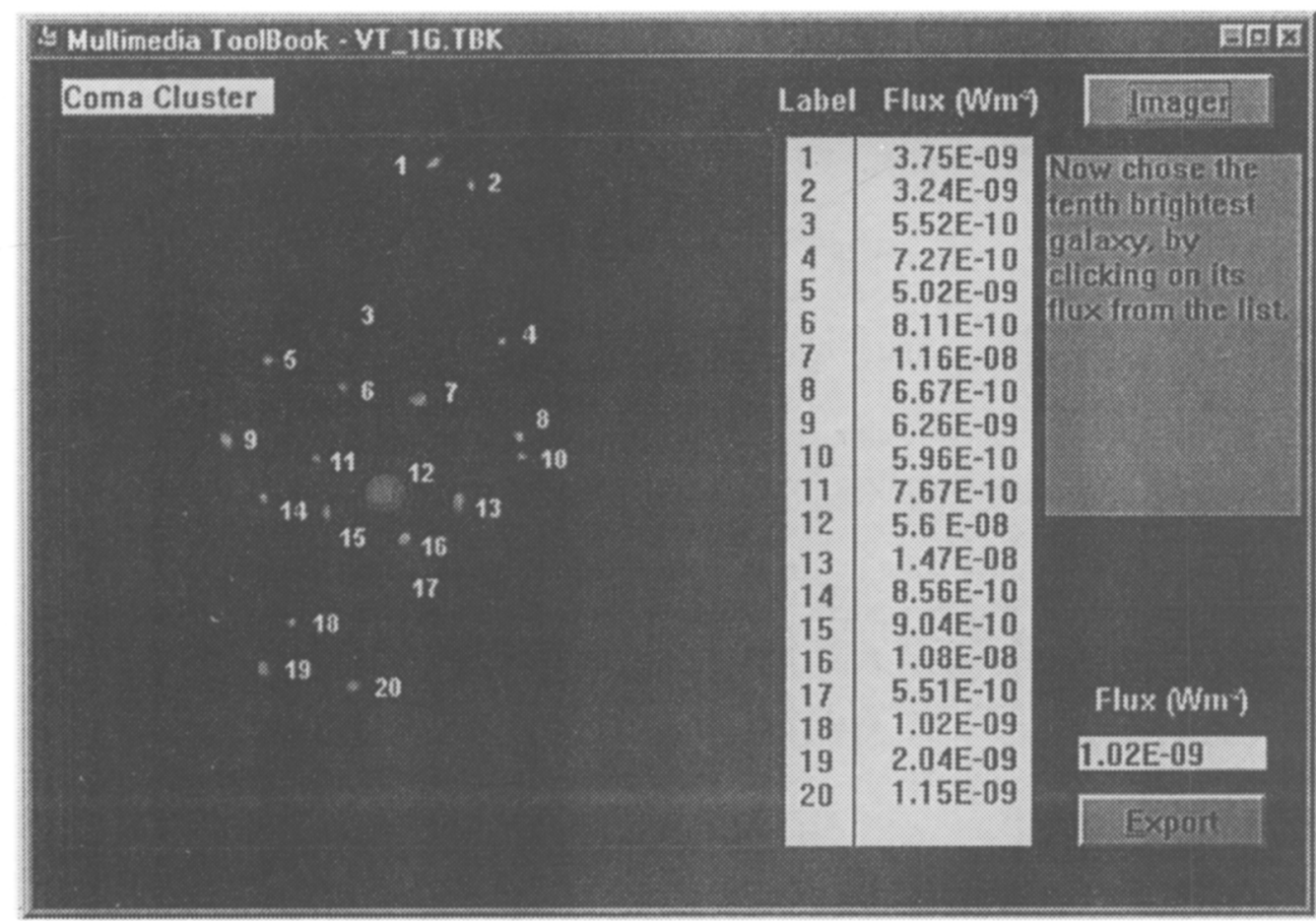

Figure 1. The imager screen from the Virtual Telescope

From a study of the text, the student brings to this exercise a knowledge of optical spectra and the measurement of speeds using a redshift. They also appreciate the inverse square law relating the brightness (in $\mathrm{W} \mathrm{m}^{-2}$ ) to the absolute luminosity of a galaxy and its distance, and an understanding of the statistical arguments behind the adoption of 'standard candles'.

\subsection{Imager}

'The package opens with a visible light image of the night sky, in which eight clusters of galaxies are highlighted. The clusters (Perseus, Coma, Hercules, Ursa Major I, Leo, Gemini, Boötes, and Hydra) are chosen to be representative and at a range of redshift between about 0.02 and 0.2 . On selecting an individual cluster, the virtual telescope provides an image of that cluster. The images used are from the digitised sky survey, available over the internet from STScI. Regions of sky about 10 arcmin square are used and the images have been digitized at a resolution of 1.6 arcsec per pixel. A typical view of the imager screen is shown in Figure 1. The student then has the choice of selecting either 'spectrometer' or 'photometer' to carry the investigation further.

\subsection{Spectrometer}

Selecting 'spectrometer' and choosing an individual galaxy within the cluster, the student obtains a spectrum of the galaxy in question between about $350 \mathrm{~nm}$ and $700 \mathrm{~nm}$. These galaxy spectra are generated internally from a template spectrum of a galaxy in the Coma cluster, adjusted for the required redshift and noise characteristics. On calling up a 'standard spectrum' (actually that of a K-type star) the student is invited to 


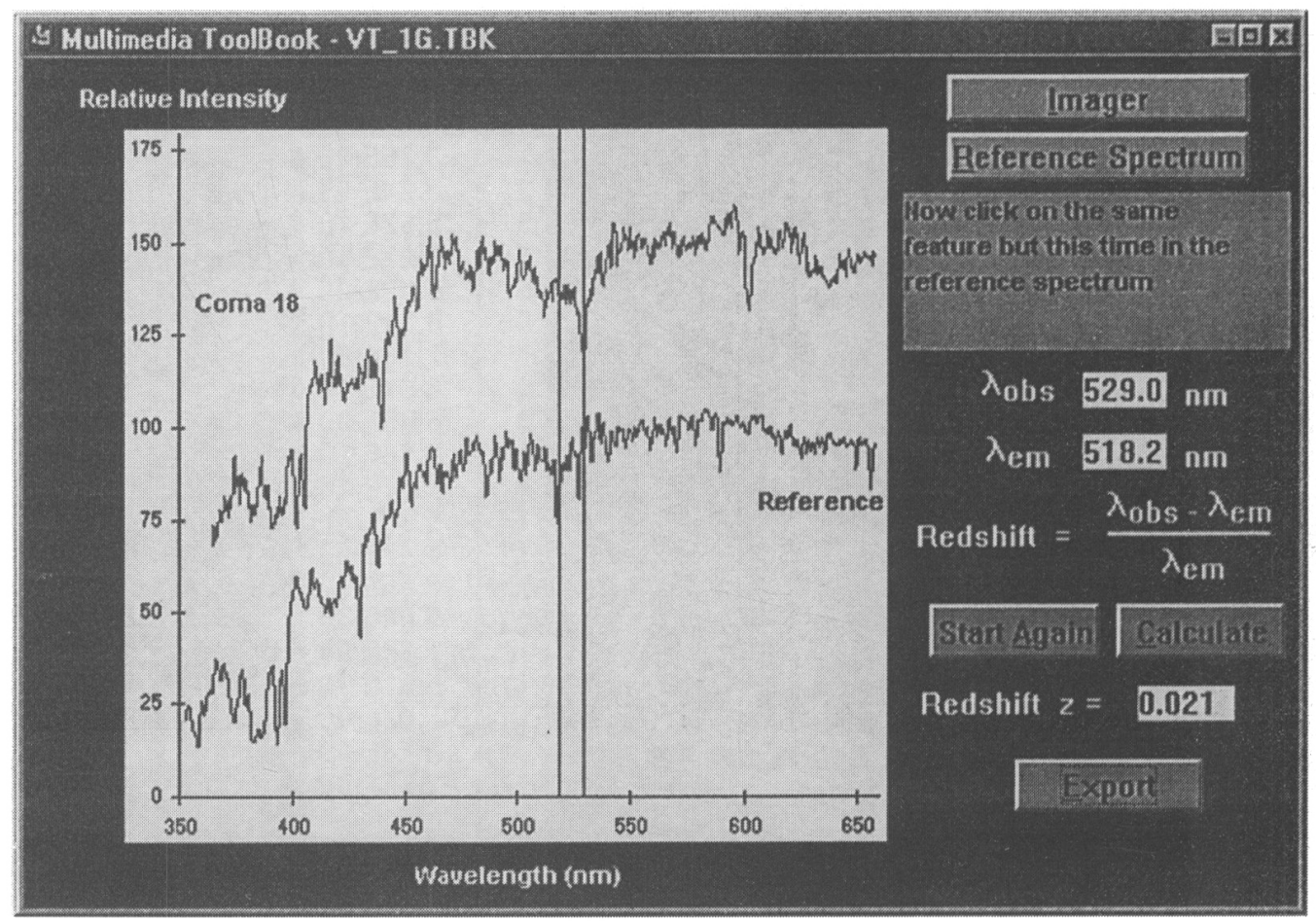

FiguRE 2. The spectrometer screen from the Virtual Telescope

identify common features in the two spectra, from which a 'redshift' is calculated. This is converted into a recession speed in $\mathrm{km} \mathrm{s}^{-1}$ and exported to the on-line notebook. The procedure may be repeated for other features in this spectrum or for spectra of other galaxies in this cluster - all of which should give similar results for the recession speed of the cluster in question. An example of the spectrometer screen is shown in Figure 2.

\subsection{Photometer}

Returning to the 'imager' screen and selecting the 'photometer' option, the virtual telescope performs aperture photometry on (up to) 20 galaxies in the cluster and identifies which galaxy has which brightness. The student selects the 10th brightest galaxy in the cluster from the information presented. By comparing the brightness with that of a standard 10th brightest galaxy at a known distance, the distance to the cluster in question is determined. This distance is converted into $\mathrm{Mpc}$ and exported to the notebook. An example of the photometer screen is shown in Figure 3.

\subsection{Results}

The whole procedure of speed and distance measurement is repeated for up to eight clusters of galaxies. The distance/speed data that have been obtained are plotted and a straight line fit to the data is found. The gradient is measured - this is the Hubble constant in $\mathrm{km} \mathrm{s}^{-1} \mathrm{Mpc}^{-1}$. The answers that the student obtains at each stage can be checked (i.e. the redshifts and distances for each galaxy, and the final values of Hubble constant). If deviant values are obtained the computer will prompt with re-try messages. 


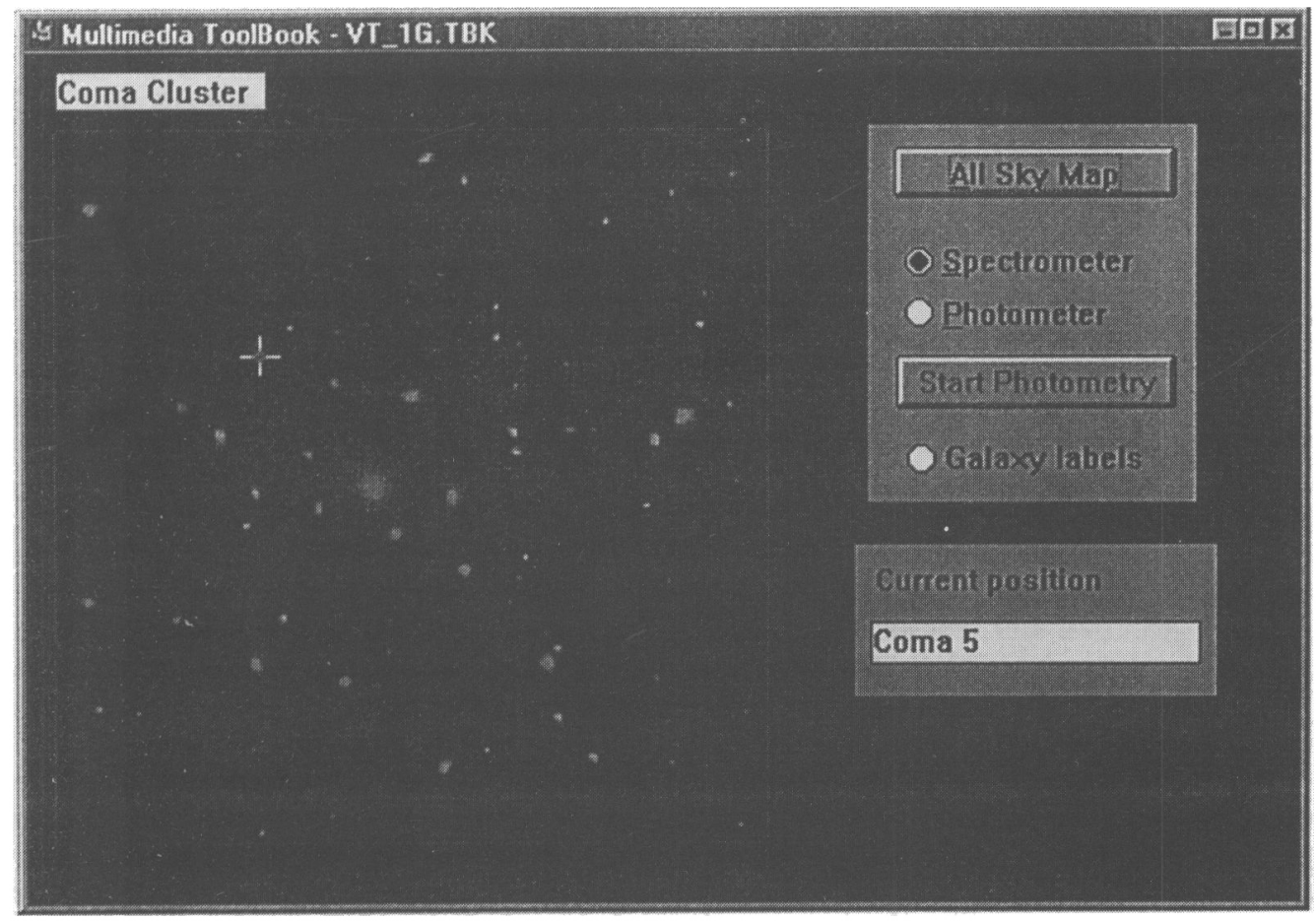

Figure 3. The photometer screen from the Virtual Telescope

\section{Conclusions}

The S103 Virtual Telescope is still under development; however, it is hoped that it will provide our students with an exciting and challenging experience of making real cosmological measurements. We hope it will demonstrate that CD-ROM based multimedia activities have an important role to play in teaching astronomy concepts as part of a distance taught, broad based, introductory science course. 